\title{
SINGULARITIES IN CONTACT GEOMETRY
}

\author{
MARC CHAPERON \\ Institut de mathématiques de Jussieu, Université Paris 7 \\ CASE 7012, 2 place Jussieu, F-75251 Paris Cedex 05 \\ E-mail: chaperon@math.jussieu.fr
}

\begin{abstract}
In the first half of the paper, we consider singularities of infinitesimal contact transformations and first order partial differential equations, the main results being related to the classical Sternberg-Chen theorem for hyperbolic germs of vector fields. The second half explains how to construct global generating phase functions for solutions of Hamilton-Jacobi equations and see what their singularities look like.
\end{abstract}

Introduction. This is a more or less self-contained (self-centred?) presentation of the subject. A contact form on a manifold $V$ is a 1-form $c$ such that, for every $x \in V$, the linear form $c_{x}$ on $T_{x} V$ is non-zero and the bilinear form on the hyperplane Ker $c_{x}$ induced by $d c_{x}$ is non-degenerate; as there is no non-degenerate alternate bilinear form on an odd-dimensional space, this implies that $\operatorname{dim} V$ is odd.

EXAmples. A generic 1-form $\alpha$ on an odd-dimensional manifold $W$ is a contact form at generic points: more precisely, there is a closed, smooth hypersurface $S$ of $W$ such that $\left.\alpha\right|_{W \backslash S}$ is a contact form.

Let $J^{1} M=J^{1}(M, \mathbf{R})$ be the space of 1 -jets $j^{1} f(x)=\left(x, f(x), d f_{x}\right)$ of real functions $f$ on a manifold $M$; if we denote the points of $J^{1} M$ by $(q, u, p), q \in M, u \in \mathbf{R}, p \in\left(T_{q} M\right)^{*}$, the canonical contact form of $J^{1} M$ is $c_{M}:=d u-p d q$. Locally, a section $\sigma$ of the "source" projection $s_{M}:(q, u, p) \mapsto q$ is of the form $j^{1} f$ if and only if $\sigma^{*} c_{M}=0$.

A contact structure on $V$ is a hyperplane field $\mathcal{K}$ (i.e. a codimension 1 vector subbundle of $T V$ ) with the following property: for each $a \in V$, there exists a contact form $c$ on a neighbourhood $U$ of $a$ such that $\mathcal{K}_{x}=\operatorname{Ker} c_{x}$ for every $x \in U$.

In the first part of the paper, we consider singularities in contact geometry proper: given a hypersurface $E$ of a manifold $V$ endowed with a contact structure $\mathcal{K}$, we explain how to describe what happens near a point $a \in E$ at which $T_{a} E=\mathcal{K}_{a}$.

In the second part of the paper, closer to our original talk, we consider "concrete" contact structures like that of $J^{1} M$, on spaces equipped with a projection like $s_{M}$; the

2000 Mathematics Subject Classification: Primary 46C20; Secondary 32G81.

The paper is in final form and no version of it will be published elsewhere. 
singularities have more to do with this projection than with the contact structure itself. All objects are assumed "smooth enough" (for example $C^{\infty}$ ).

Acknowledgements. I wish to thank the organisers of this very pleasant and instructive meeting for their hospitality.

\section{Some background; singularities of Lie and Liouville fields}

1.1. Lie fields. Given a contact structure $\mathcal{K}$ on a manifold $V$, a contact transformation of $\mathcal{K}$ is a transformation $h$ of $V$ which preserves $\mathcal{K}$ : for each $x \in V$, the derivative $T_{x} h$ sends $\mathcal{K}_{x}$ onto $\mathcal{K}_{h(x)}$. A Lie field of $\mathcal{K}$ is an infinitesimal contact transformation, i.e. a vector field $X$ on $V$ whose flow consists of contact transformations. For each contact form $c$ generating $\mathcal{K}$ in an open subset of $V$, this is expressed by the identity $c \wedge \mathcal{L}_{X} c=0$, where $\mathcal{L}_{X}$ is the Lie derivative with respect to $X$.

Proposition 1 (Lie). If $\mathcal{K}$ is the contact structure generated by a contact form $c$ on $V$, then $X \mapsto c X$ is an isomorphism of the space of Lie fields of $\mathcal{K}$ onto the space of real functions on $V$. Hence each Lie field is determined by its (contact) Hamiltonian $-c X$ with respect to $c$.

Proof. Given a real function $H$ on $V$, we wish to show that there is a unique Lie field $X$ such that

$$
H=-c X
$$

By the Cartans' homotopy formula $\mathcal{L}_{X} c=d(c X)+(d c) X$ (interior product), this is expressed by (1) and

$$
(-d H+(d c) X) \wedge c=0
$$

Now, for each $x \in V$, we have $T_{x} V=\mathcal{K}_{x} \oplus \operatorname{Ker} d c_{x}$. If we write $X_{x}=Y_{x}+Z_{x}$ in this decomposition, (1) reads $c_{x} Z_{x}=-H(x)$, which determines $Z_{x}$ since $c_{x}$ is a linear coordinate on the one-dimensional kernel of $d c_{x}$. Moreover, at $x,(2)$ can be written

$$
\left.\left(-d H_{x}+d c_{x} Y_{x}\right)\right|_{\mathcal{K}_{x}}=0,
$$

which determines $Y_{x}$ because $\left.v \mapsto\left(d c_{x} v\right)\right|_{\mathcal{K}_{x}}$ is an isomorphism of $\mathcal{K}_{x}$ onto its dual space.

1.2. First order partial differential equations and such. Under those hypotheses, let $E$ be a hypersurface of $V$. Locally, the contact structure is defined by a 1 -form $c$ and $E$ is the set of zeros of a real function $H$, which is the contact Hamiltonian of a unique Lie field $X$ with respect to $c$. Now, we have $X_{x} \in \mathcal{K}_{x} \cap T_{x} E$ for every $x \in E$; indeed, (1) yields $c_{x} X_{x}=-H(x)=0$, hence $\left(-d H_{x}+d c_{x} X_{x}\right) X_{x}=0$ by (3), i.e. $d H_{x} X_{x}=0$ since $d c_{x}\left(X_{x}, X_{x}\right)=0$. Of course $X$ depends on the choice of $H$ and $c$, but, for each $x \in E$, the subspace generated by $X_{x}$ depends only on $E$ and $\mathcal{K}$ : indeed, either $T_{x} E=\mathcal{K}_{x}$, in which case $X_{x}=0$ because of (3), or $T_{x} E \cap \mathcal{K}_{x}$ is a hyperplane of $\mathcal{K}_{x}$, whose orthogonal for the non-degenerate form $\left.d c_{x}\right|_{\mathcal{K}_{x} \times \mathcal{K}_{x}}$ is the line $\chi_{x}$ generated by $X_{x}$, again by (3). Note that this does not depend on the choice of $c$, for another choice would replace $c$ by $\lambda c$ for some non-vanishing real function $\lambda$, hence the restriction of $d(\lambda c)_{x}=\lambda(x) d c_{x}+d \lambda_{x} \wedge c_{x}$ to $\mathcal{K}_{x} \times \mathcal{K}_{x}$ would be $\left.\lambda(x) d c_{x}\right|_{\mathcal{K}_{x} \times \mathcal{K}_{x}}$. 
In summary, $E$ consists of the closed subset $\Sigma$ of those $x$ such that $T_{x} E=\mathcal{K}_{x}$ (called singular points and generically isolated) and the open subset $E \backslash \Sigma$, on which there is a field of lines $x \mapsto \chi_{x} \subset T_{x} E \cap \mathcal{K}_{x}$ called characteristic lines; the integral curves of this line field are the characteristic curves.

First order partial differential equations and their solutions. A first order partial differential equation on an $n$-dimensional manifold $M$ is an equation of the form $H\left(q, u, \frac{d u}{d q}\right)=0$, where $H$ is a real function on $J^{1} M$. A solution of the equation is a function $\varphi$ defined on some open subset of $M$ and such that $H \circ j^{1} \varphi=0$. If we identify $\varphi$ to the image of the section $j^{1} \varphi$ of $s_{M}$, we see that a solution of the equation is a Legendrian submanifold, i.e. an $n$-dimensional submanifold on which $c_{M}$ induces the null 1-form, or, equivalently, an $n$-dimensional submanifold all of whose tangent spaces are contained in the corresponding contact hyperplanes.

Hence, forgetting about the projection $s_{M}$, we can define a geometric solution of the equation to be a Legendre submanifold of $J^{1} M$ contained in $H^{-1}(0)$. Such a generalised solution may project badly onto $M$; the second half of this paper provides scissors allowing the construction of a "normal" solution from a geometric one.

Generalised first order partial differential equations. If we go back to the more general situation of an arbitrary contact structure $\mathcal{K}$ on a manifold $V$ of dimension $2 n+1$, a generalised first order partial differential equation is just a hypersurface $E$ of $V$; its solutions are the Legendrian submanifolds of $V$ contained in $E$.

EXAMPLE. If $n=1$, the (maximal) solutions contained in $E \backslash \Sigma$ are the characteristic curves (which can sometimes be extended smoothly through singular points).

1.3. Cauchy problems. We shall prove that first order partial differential equations have solutions and explain how those solutions can be constructed.

Classical Cauchy problems. First consider a Hamilton-Jacobi equation on $M$, i.e. a first order partial differential equation on $I \times M$ of the form $\partial_{t} u+H\left(t, q, u, \partial_{q} u\right)=0$ for some interval $I \ni 0$ and some real function $H$ on $I \times J^{1} M$, where $t, q$ denote the variables in $I, M$ respectively. A Cauchy problem consists in looking for solutions $\varphi(t, q)$ of the equation which take a prescribed value $\varphi_{0}(q)$ for $t=0$.

Denote the points of $J^{1}(I \times M)$ by $(t, q, u, \tau, p)$ with $(t, q) \in I \times M, u \in \mathbf{R}$ and $(\tau, p) \in$ $\left(T_{(t, q)}(I \times M)\right)^{*}=\mathbf{R} \times\left(T_{q} M\right)^{*}$, hence $c_{I \times M}=d u-\tau d t-p d q$. Our Cauchy problem can be reformulated as follows: find a Legendre submanifold $L$ (the image of $j^{1} \varphi$ ) contained in the hypersurface $\tau+H(t, q, u, p)=0$ and passing through the $(n-1)$-dimensional submanifold $L_{0}$ of $J^{1}(I \times M)$ defined by $(q, u, p)=j^{1} \varphi_{0}(q)$ and $\tau=-H\left(t, j^{1} \varphi_{0}(q)\right)$. This is not absurd since $L_{0}$ obviously is isotropic, i.e. $c_{I \times M}$ induces the zero form on $L_{0}$ or, equivalently, $L_{0}$ is everywhere tangent to the contact structure.

Generalised Cauchy problems. Let $E$ be a generalised first order partial differential equation in a $(2 n+1)$-dimensional manifold $V$ endowed with a contact structure $\mathcal{K}$; a generalised Cauchy problem for the equation consists in looking for the solutions of $E$ which contain a given isotropic $(n-1)$-dimensional submanifold $L_{0}$ of $V$. The problem is well-posed if, for each $x \in L_{0}$, the characteristic line $\chi_{x}$ exists and does not lie in $T_{x} L_{0}$. 
ExAmple. A classical Cauchy problem is well-posed: indeed, with the notation of the definition, the $t$-component of the Lie field on $J^{1}(I \times M)$ generated by the Hamiltonian $\tau+H(t, q, u, p)$ is 1 .

Proposition 2. Let $L_{0} \subset E$ be a well-posed generalised Cauchy problem. Then, a solution $L$ of this Cauchy problem can be obtained by taking the union of local characteristic curves through points of $L_{0}$. Moreover, any solution of the given Cauchy problem coincides with $L$ near $L_{0}$, for every solution of $E$ is a union of characteristic curves.

Proof. Our hypothesis clearly implies that $L$ is an $n$-dimensional submanifold near $L_{0}$. As the problem is local, we may assume that $\mathcal{K}$ is defined by a contact form $c$ and that $E$ is the set of zeros of a real function $H$ having 0 as a regular value; if $g^{t}$ denotes the flow of the Lie field $X$ whose Hamiltonian is $H$, the submanifold $L$ consists (locally) of all points $g^{t}(x)$ with $x \in L_{0}$ and $t$ small enough, and its tangent space at $g^{t}(x)$ is the direct sum of $T_{x} g^{t}\left(T_{x} L_{0}\right)$ and $\mathbf{R} T_{x} g^{t}\left(X_{x}\right)=\mathbf{R} X_{g^{t}(x)}$; our first assertion follows because both of these subspaces are contained in $\mathcal{K}_{g^{t}(x)}$, the first because $g^{t}$ is a contact transformation and $L_{0}$ is isotropic, the second because $c_{g^{t}(x)} X_{g^{t}(x)}=-H\left(g^{t}(x)\right)=0$ (recall that $x \in E$ implies $g^{t}(x) \in E$ since $X$ is tangent to $\left.E\right)$.

Given a solution $S$ of $E$, we have to show that it is the union of characteristic curves, i.e. that we have $\chi_{x} \subset T_{x} S$ for every $x \in S$. Assume that, for some $a \in S$, we have $\chi_{a} \not \subset T_{a} S$; then, what we have just done for $L_{0}$ applies to $S$ and shows that, near $a$, some union of characteristic curves through points of $S$ is an $(n+1)$-dimensional isotropic submanifold of $V$, contradicting the following

BASIC FACT. An isotropic submanifold $V$ of a $(2 n+1)$-dimensional contact manifold has dimension at most $n$.

Proof. The problem being local, we may assume that the contact structure $\mathcal{K}$ is defined by a contact form $c$. If we denote by $i$ the inclusion of $L_{0}$, the relation $i^{*} c=0$ yields $d\left(i^{*} c\right)=0$, i.e. $i^{*} d c=0$. Hence, for each $x \in V$, the subspace $T_{x} V$ is contained in $\mathcal{K}_{x}$ and $\left.d c_{x}\right|_{T_{x} V \times T_{x} V}=0$; this last equation means that $T_{x} V$ is contained in its orthogonal $T_{x} V^{\perp}$ for the non-degenerate bilinear form $\left.d c_{x}\right|_{\mathcal{K}_{x} \times K_{x}}$; hence we have $\operatorname{dim} T_{x} V \leq \operatorname{dim} T_{x} V^{\perp}=$ $2 n-\operatorname{dim} T_{x} V$.

REMARK. The generic singularities of solutions of ill-posed generalised Cauchy problems can be (and therefore must have been) studied; it should be noted that, in the classical case of $J^{1} M$, their images by the projection $(q, u, p) \mapsto(q, u)$ onto $J^{0} M=M \times \mathbf{R}$ are more singular than the generic wavefronts, which are the projections of non-singular Legendre submanifolds.

Corollary 3. A smooth classical solution $\varphi:(t, q) \mapsto \varphi_{t}(q)$ of a classical Cauchy problem must be as follows near $t=0$ : with the notation of the definition, the Legendre submanifold $j^{1} \varphi_{t}(M)$ is the image of $j^{1} \varphi_{0}(M)$ by the contact transformation $g_{t}$ of $J^{1} M$ obtained by integrating $\left({ }^{1}\right)$ the time-dependent Lie field $X_{t}$ whose Hamiltonian is $H_{t}:(q, u, p) \mapsto H(t, q, u, p)$.

$\left({ }^{1}\right)$ Meaning that $g_{0}(x)=x$ and $\partial_{t} g_{t}(x)=X_{t}\left(g_{t}(x)\right)$. 
Proof. As mentioned before, the $t$-component of the Lie field on $J^{1}(I \times M)$ generated by the Hamiltonian $\tau+H(t, q, u, p)$ is 1 ; as its $(q, p)$-component is $X_{t}(q, p)$, its flow $\tilde{g}^{s}:(t, q, u, \tau, p) \mapsto\left(t^{s}, q^{s}, u^{s}, \tau^{s}, p^{s}\right)$ is given by $t^{s}=t+s,\left(q^{s}, u^{s}, p^{s}\right)=g_{s} \circ g_{t}^{-1}(q, u, p)$ and (since it preserves the zero level of its Hamiltonian) $\tau^{s}=-H\left(t^{s}, q^{s}, u^{s}, p^{s}\right)$ if $\tau=$ $-H(t, q, u, p)$; the geometric solution of the equation satisfying the given initial condition being (locally) the union of all $\tilde{g}^{t}\left(0, q, \varphi_{0}(q),-H\left(0, j^{1} \varphi_{0}(q)\right), d \varphi_{0}(q)\right)$, this implies what we claimed.

Note. There are two difficulties with this corollary: $g_{t}\left(j^{1} \varphi_{0}(M)\right)$ may not be defined for all $t$ and, even if it is, $g_{t}\left(j^{1} \varphi_{0}(M)\right)$ can be badly projected onto $M$ for some $t$ 's (if $M$ is not compact, this can even be the case for every $t \neq 0$ ). One cannot do much against the first difficulty, but the second can sometimes be settled by constructing a genuine (weak) solution of the Cauchy problem from the badly projected geometric solution, as we shall see in part 2.

1.4. Singularities of Lie fields and first order equations. Given a hypersurface $E$ of a $(2 n+1)$-dimensional manifold $V$ endowed with a contact structure $\mathcal{K}$, consider a singular point of the generalised first order partial differential equation $E$, namely an $a \in E$ such that $T_{a} E=\mathcal{K}_{a}$, which can also be expressed as follows: if $c$ is a contact form defining $\mathcal{K}$ near $a$ and $H$ a real function having 0 as a regular value and such that $H^{-1}(0)=E$ near $a$, the Lie field $X$ whose Hamiltonian with respect to $c$ is $H$ vanishes at $a$.

Thus, the classification of (generalised) first order partial differential equations near their singular points has to do with the classification of Lie fields near their zeros. Now, transversality arguments show that (Baire-)almost every Lie field has only hyperbolic zeros $\left({ }^{2}\right)$, and we have the following contact version of the Sternberg-Chen theorem, essentially due to Guillemin and Schaeffer:

TheOrem 4. For $j=0,1$, let $\mathcal{K}_{j}$ be a contact structure on a manifold $V_{j}$ and let $X_{j}$ be germ at $a_{j} \in V_{j}$ of a Lie field having a hyperbolic zero at $a_{j}$. The following two conditions are equivalent:

i) The Lie fields $X_{0}$ and $X_{1}$ are contact conjugate: there is a local contact transformation $\left(^{3}\right) h:\left(V_{0}, a_{0}\right) \rightarrow\left(V_{1}, a_{1}\right)$ such that $h^{*} X_{1}=X_{0}$.

ii) $X_{0}$ and $X_{1}$ are formally contact conjugate: there exists a local contact transformation $g:\left(V_{0}, a_{0}\right) \rightarrow\left(V_{1}, a_{1}\right)$ such that $g^{*} X_{1}$ and $X_{0}$ have infinite contact at $a_{0}$.

Idea of a proof. Of course i) implies ii). Assuming ii), we may replace $X_{1}$ by $g^{*} X_{1}$ and assume that $X_{0}, X_{1}$ are germs of Lie fields having infinite contact at 0 . If we denote their flows by $\rho_{0}^{t}, \rho_{1}^{t}$ and the stable (resp. unstable) manifold of $X_{0}$ by $W^{s}$ (resp. $W^{u}$ ), two cases may occur:

If $X_{0}$ is in the Poincare domain, i.e. the germ of $V_{0}$ at $a_{0}$ is $W^{s}$ or $W^{u}$, then it is comparatively easy to show that $\rho_{1}^{-t} \circ \rho_{0}^{t}$ (in the attracting case) or $\rho_{1}^{t} \circ \rho_{0}^{-t}$ (in the repelling case) converges in the $C^{\infty}$ sense when $t \rightarrow+\infty$ to the required $h$, which is a contact transformation like every $\rho_{1}^{-t} \circ \rho_{0}^{t}$ and a conjugacy because $\rho_{1}^{-t} \circ \rho_{0}^{t}\left(\rho_{0}^{s}(x)\right)=$ $\rho_{1}^{s}\left(\rho_{1}^{-t-s} \circ \rho_{0}^{t+s}(x)\right)$, hence at the limit $h\left(\rho_{0}^{s}(x)\right)=\rho_{1}^{s}(h(x))$.

$\left({ }^{2}\right)$ Meaning that none of the eigenvalues of its differential at a zero lies on the imaginary axis.

$\left({ }^{3}\right)$ Meaning that $T_{x} h\left(\mathcal{K}_{0}\right)_{x}=\left(\mathcal{K}_{1}\right)_{h(x)}$ for every $x$. 
Otherwise, $X_{0}$ is in the Siegel domain and similar arguments show that the infinite jets $\left.\left(j^{\infty}\left(\rho_{1}^{-t} \circ \rho_{0}^{t}\right)\right)\right|_{W^{s}}$ and $\left.\left(j^{\infty}\left(\rho_{1}^{t} \circ \rho_{0}^{-t}\right)\right)\right|_{W^{u}}$ converge when $t \rightarrow+\infty$; their limits define the jet along $W^{s} \cup W^{u}$ of a diffeomorphism $h_{1}$ having infinite contact with the identity at $a_{0}$, such that $h_{1}^{*} X_{1}$ and $X_{0}$ have infinite contact along $W^{s} \cup W^{u}$; moreover, so have $h_{1}^{*} \mathcal{K}_{0}$ and $\mathcal{K}_{0}$.

Then, one can define a local conjugacy $h_{2}$ between $X_{0}$ and $h_{1}^{*} X_{1}$ as the solution of a "Cauchy problem": take a small embedded sphere $S$ around $a_{0}$ in $W^{s}$, transversal to the orbits of $X_{0}$, choose any germ $\tilde{S}$ at $S$ of a hypersurface transversal to $W^{s}$, and decide that $h_{2}(x)=x$ for $x \in \tilde{S}$; if $\rho_{2}^{t}$ denotes the flow of $h_{1}^{*} X_{1}$, our conjugacy is given for $x \notin W^{u}$ by $h_{2}(x)=\rho_{2}^{\tau(x)}\left(\rho_{0}^{-\tau(x)}(x)\right)$, where $\tau(x)$ is determined by the condition $\rho_{0}^{-\tau(x)}(x) \in \tilde{S}$. It can be shown that this extends by continuity to a local diffeomorphism $h_{2}:\left(V_{0}, a_{0}\right) \rightarrow\left(V_{0}, a_{0}\right)$ having infinite contact with the identity along $W^{s} \cup W^{u}$.

Now, we do have $h_{2}^{*} h_{1}^{*} X_{1}=X_{0}$ but not $h_{2}^{*} h_{1}^{*} \mathcal{K}_{1}=\mathcal{K}_{0}$, so we need a third (fourth) diffeomorphism germ $h_{3}$ preserving $X_{0}$ and pulling back $h_{2}^{*} h_{1}^{*} \mathcal{K}_{1}$ to $\mathcal{K}_{0}$; this will prove our theorem with $h=h_{1} \circ h_{2} \circ h_{3}$ (or, rather, $h=g \circ h_{1} \circ h_{2} \circ h_{3}$ ). To do so, we first work in the (local) quotient $Q$ of $V_{0} \backslash W^{u}$ by $\rho_{0}^{1}$, which is a thickened version $\tilde{S} \times \mathbf{T}$ (where $\mathbf{T}=\mathbf{R} / \mathbf{Z}$ ) of the corresponding quotient $\tilde{S}$ by the flow $\rho_{0}^{t}$. The two contact structures on $Q$ induced by $\mathcal{K}_{0}$ and $h_{2}^{*} h_{1}^{*} \mathcal{K}_{1}$ have infinite contact along the image $Q^{s}$ of $W^{s}$ and are invariant by the $\mathbf{T}$-action induced by the flow $\rho_{0}^{t}$. Hence an equivariant (local) version of Gray's theorem enables us to find a diffeomorphism germ $\left(Q, Q^{s}\right) \rightarrow\left(Q, Q^{s}\right)$ commuting to the T-action, having infinite contact with the identity along $Q_{s}$ and pulling back the second contact structure to the first. This diffeomorphism germ can be lifted in a unique fashion to a diffeomorphism germ having infinite contact with the identity along $W^{s} \backslash\left\{a_{0}\right\}$, and the same methods as in the previous step show that the lifted diffeomorphism can be extended by the identity on $W^{u}$ so as to get $h_{3}$.

Notes. The interested reader can find the relevant details in [6], [8]. Of course, forgetting about the contact structure, we get a proof of the Sternberg-Chen theorem.

In the Poincaré domain, the result is much easier and more effective, since there exists an integer $k$, depending only on the differential $d X_{0}(a)$, such that $X_{1}$ and $X_{0}$ are conjugate if and only if they are formally conjugate at order $k$. Moreover, this remains true in the analytic category, which is far from being the case in the Siegel domain because of "small divisors".

Recently, the author has put all such results in the framework of invariant manifold theory [13], [14]. The initial remark is that $h$ is a conjugacy between $X_{0}$ and $X_{1}$ if and only if the local vector field $X_{0} \times X_{1}:\left(x_{0}, x_{1}\right) \mapsto\left(X_{0}\left(x_{0}\right), X_{1}\left(x_{1}\right)\right)$ on $V_{0} \times V_{1}$ is tangent to the graph of $h$.

The above rather abstract statement of the theorem should be completed with "concrete" normal forms. The first step is the following classical result:

Proposition 5 (Darboux). For every point a of a $(2 n+1)$-dimensional manifold $V$ endowed with a contact form $c$, there exists a local diffeomorphism $h:(V, a) \rightarrow\left(J^{1} \mathbf{R}^{n}, 0\right)$ such that $h_{*} c=c_{\mathbf{R}^{n}}$. In other words, up to smooth changes of coordinates, there is only one local contact form in each odd dimension. 
The theory of formal normal forms can then be obtained as follows (we refer to [8] for details): write $c_{\mathbf{R}^{n}}=d w+\frac{1}{2}(q d p-p d q)$, where $w:=u+\frac{1}{2} p q$, identify each Lie field to its Hamiltonian and the Lie bracket of two Lie fields to the Lagrange bracket of their Hamiltonians. This makes the space $\hat{\mathcal{E}}^{\infty}$ of formal power series in the variables $w, q, p$ into a Lie algebra, on which the Lagrange bracket respects the graduation obtained by giving weight 2 to $w$ and weight 1 to $q$ and $p$; for each integer $k$, the (finite-dimensional) space $\hat{\mathcal{E}}^{k}$, obtained by taking the quotient of $\hat{\mathcal{E}}^{\infty}$ by its elements of order $>k$ (for this graduation) is made into a Lie algebra; a formal Lie field $\hat{X}^{\infty}$ vanishing at 0 identifies to an infinitesimal automorphism of $\hat{\mathcal{E}}^{\infty}$, and it can be shown that its semi-simple part $\hat{\sigma}^{\infty}$, obtained by taking the projective limits of the semi-simple parts of the automorphisms of the $\hat{\mathcal{E}}^{k}$ 's induced by $\hat{X}^{\infty}$, is (induced by) a formal Lie field, which is linearisable (this is the reason for preferring $w$ to $u$ ) in the following sense: there exists a formal contact transformation $\hat{h}$ sending $\hat{\sigma}^{\infty}$ onto its linear part (in terms of Hamiltonians, its homogeneous part of "degree" 2). Then, the image by $h$ of the nilpotent (i.e. nilpotent in each $\hat{\mathcal{E}}^{k}$ with $k<\infty$ ) part $\hat{\nu}^{\infty}$ of $\hat{X}^{\infty}$ is a formal Lie field (or formal Hamiltonian) commuting with $\hat{\sigma}^{\infty}$; hence $\hat{h}_{*} \hat{\nu}^{\infty}$ commutes with $\hat{h}_{*} \hat{\sigma}^{\infty}$ and therefore consists of "resonant" terms.

Then, one has a look at this formal normal form $\hat{h}_{*} \hat{\sigma}^{\infty}+\hat{h}_{*} \hat{\nu}^{\infty}$ : if it is polynomial, it can be identified to a "concrete" normal form; otherwise, it can be expressed (in a non-unique way in general) as a formal power series in finitely many "resonant monomials", and a corresponding "concrete" normal form is just any smooth function of those monomials whose Taylor expansion at 0 is this formal power series. By Theorem 4 above, every hyperbolic germ of a Lie field admits such a normal form.

When the Hamiltonian $H$ of a Lie field is put into normal form, so is $\left({ }^{4}\right)$ the first order partial differential equation $H=0$. However, if one is interested in the equation and not in $H$ itself, there is a better, easier way which we shall now explain.

1.5. Singularities of first order equations and Liouville fields. Given a generalised first order partial differential equation, i.e. a hypersurface $E$ of a $(2 n+1)$-dimensional manifold $V$ endowed with a contact structure $\mathcal{K}$, and an $a \in E$ such that $T_{a} E=\mathcal{K}_{a}$, Proposition 5 above enables us to assume that $E=J^{1} \mathbf{R}^{n}, a=0$ and that $\mathcal{K}$ is the standard contact structure $d u=p d q$; if $H:\left(J^{1} \mathbf{R}^{n}, 0\right) \rightarrow \mathbf{R}$ is a local submersion such that $E=H^{-1}(0)$ near 0 , the relation $T_{a} E=\mathcal{K}_{a}$ reads $d H(0) \wedge d u=0$; as we have $d H(0) \neq 0$, the implicit function theorem implies that, near $0, E$ is the graph of a function $u=g(q, p)$ with $g(0)=0$ and $d g(0)=0$. Taking $(q, p)$ as coordinates on $E$, we can see that its characteristics are the flowlines of the vector field

$$
X_{g}:(q, p) \mapsto\left(\partial_{p} g(q, p),-\partial_{q} g(q, p)+p\right),
$$

which is the sum of the Hamiltonian vector field $\overrightarrow{d g}:(q, p) \mapsto\left(\partial_{p} g(q, p),-\partial_{q} g(q, p)\right)$ with Hamiltonian $g$ and the "standard Liouville field" $X_{0}:(q, p) \mapsto(0, p)$. It follows that $X_{g}$ is a Liouville vector field, meaning that $\mathcal{L}_{X_{g}} \omega_{\mathbf{R}^{n}}=\omega_{\mathbf{R}^{n}}$, where $\omega_{\mathbf{R}^{n}}=d q \wedge d p$ is the standard symplectic form of $T^{*} \mathbf{R}^{n}=\mathbf{R}^{n} \times \mathbf{R}^{n *}$; every local Liouville field vanishing at 0 is of that form, and the following result is easy:

$\left({ }^{4}\right)$ Forgetting about the projection $(q, u, p) \mapsto(q, u)$, but a contact transformation $h$ sends the geometric solutions of an equation $E$ onto the geometric solutions of $h(E)$. 
Lemma 6. Given two function germs $g_{0}, g_{1}:\left(T^{*} \mathbf{R}^{n}, 0\right) \rightarrow(\mathbf{R}, 0)$ with $d g_{0}(0)=$ $d g_{1}(0)=0$, the following two conditions are equivalent:

1. There exists a diffeomorphism germ $\tilde{h}:\left(J^{1} \mathbf{R}^{n}, 0\right) \rightarrow\left(J^{1} \mathbf{R}^{n}, 0\right)$ preserving $c_{\mathbf{R}^{n}}$ and sending the graph of $g_{0}$ onto the graph of $g_{1}$.

2. There exists a diffeomorphism germ $h:\left(T^{*} \mathbf{R}^{n}, 0\right) \rightarrow\left(T^{*} \mathbf{R}^{n}, 0\right)$ preserving $\omega_{\mathbf{R}^{n}}$ and sending $X_{g_{0}}$ onto $X_{g_{1}}$.

This reduces the local classification of first order partial differential equations to that of Liouville fields. As before, there is a formal aspect (for which we refer to [26]) and an abstract part, analogous to Theorem 4, which turns out to be much simpler:

Theorem 7 ([26], [12]). For $j=0,1$, let $X_{j}$ be germ at $0 \in T^{*} \mathbf{R}^{n}$ of a Liouville field having a hyperbolic zero at 0 . The following two conditions are equivalent:

1. The Liouville fields $X_{0}$ and $X_{1}$ are symplectically conjugate: there is a local diffeomorphism $h:\left(T^{*} \mathbf{R}^{n}, 0\right) \rightarrow\left(T^{*} \mathbf{R}^{n}, 0\right)$ preserving $\omega_{\mathbf{R}^{n}}$ and such that $h^{*} X_{1}=X_{0}$.

2. There exists a local diffeomorphism $g:\left(T^{*} \mathbf{R}^{n}, 0\right) \rightarrow\left(T^{*} \mathbf{R}^{n}, 0\right)$ such that $g^{*} X_{1}$ and $X_{0}$ have infinite contact at 0 and that $g^{*} \omega_{\mathbf{R}^{n}}-\omega_{\mathbf{R}^{n}}$ vanishes at 0 .

Proof (see [12] for more details). Clearly, 1 implies 2. If 2 is satisfied, by the SternbergChen theorem, there exists a diffeomorphism germ $h_{2}:\left(T^{*} \mathbf{R}^{n}, 0\right) \rightarrow\left(T^{*} \mathbf{R}^{n}, 0\right)$, having infinite contact with $g$ at 0 , such that $h_{2}^{*} X_{1}=X_{0}$; hence $\omega_{1}:=h_{2}^{*} \omega_{\mathbf{R}^{n}}$ and $\omega_{0}:=\omega_{\mathbf{R}^{n}}$ coincide at 0 , and we should find a diffeomorphism germ $h_{1}:\left(T^{*} \mathbf{R}^{n}, 0\right) \rightarrow\left(T^{*} \mathbf{R}^{n}, 0\right)$ preserving $X_{0}$ and such that $h_{1}^{*} \omega_{1}=\omega_{0}$ : property 1 will follow with $h:=h_{2} \circ h_{1}$.

As in [28], this is done most easily by the "path method", so fundamental in singularity theory: setting $\omega_{t}:=\omega_{0}+t\left(\omega_{1}-\omega_{0}\right)$, we look for a local isotopy $\left(h_{t}\right)_{0 \leq t \leq 1}$ (where $h_{0}(z) \equiv z$ and $\left.h_{t}(0) \equiv 0\right)$ such that $h_{t}^{*} \omega_{t}=\omega_{0}$ for every $t$; such a local isotopy is obtained by integrating a time-dependent local vector field $\left(Y_{t}\right)_{0 \leq t \leq 1}$ (namely $\frac{d}{d t} h_{t}=Y_{t} \circ h_{t}$ ), and the relation $h_{t}^{*} \omega_{t} \equiv \omega_{0}$ is equivalent to $\frac{d}{d t} h_{t}^{*} \omega_{t} \equiv 0$, i.e. $\mathcal{L}_{Y_{t}} \omega_{t}+\omega_{1}-\omega_{0} \equiv 0$. Now, since $\omega_{t}$ is closed, we have $\mathcal{L}_{Y_{t}} \omega_{t}=d\left(\omega_{t} Y_{t}\right)$; moreover, for $j=0,1$, as $X_{0}$ is a Liouville field for $\omega_{j}$, we have $\omega_{j}=d\left(\omega_{j} X_{0}\right)$, hence the equation $\mathcal{L}_{Y_{t}} \omega_{t}+\omega_{1}-\omega_{0} \equiv 0$ reads $d\left(\omega_{t} Y_{t}+\left(\omega_{1}-\omega_{0}\right) X_{0}\right) \equiv 0$. Each $\omega_{t}$ being non-degenerate near 0 because $\omega_{t}(0) \equiv \omega_{0}(0)$, we can define the required $Y_{t}$ by $\omega_{t} Y_{t}+\left(\omega_{1}-\omega_{0}\right) X_{0} \equiv 0$; it does satisfy $Y_{t}(0) \equiv 0$, and it is quite easy to check that the local isotopy $\left(h_{t}\right)$ thus generated preserves $X_{0}$.

Notes. The normal forms of Liouville fields obtained via the Jordan decomposition may be unsuitable, e.g. if one is interested in getting normal forms for pseudo-differential operators up to conjugacy by a Fourier integral operator $\left({ }^{5}\right)$ and wishes the model to be a second order differential operator; to realise this wish, it may be necessary to use "bad" normal forms as in [17].

For $n=1$ (case considered in [17]), as noticed by Davydov, it is possible not to forget about the projection $J^{1} \mathbf{R}^{n} \ni(q, u, p) \mapsto(q, u) \in J^{0} \mathbf{R}^{n}$ and get polynomial normal forms [26], [17] of first order implicit differential equations up to contact transformations preserving that projection, i.e. induced by local diffeomorphisms of $J^{0} \mathbf{R}^{n}$.

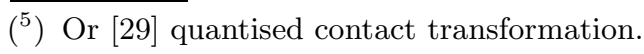


2. Some global results. The first three paragraphs of this section, essentially symplectic, are developed more or less independently from the first section. In the end, we explain how to use them in contact geometry.

2.1. Generating functions. If we still denote by $\mathbf{R}^{n *}$ the dual space of $\mathbf{R}^{n}$ and by $(q, p)$ the points of $T^{*} \mathbf{R}^{n}=\mathbf{R}^{n} \times \mathbf{R}^{n *}$, Poincaré's lemma asserts that a mapping $f$ of $\mathbf{R}^{n}$ into $\mathbf{R}^{n *}$ is the differential of a function $\varphi: \mathbf{R}^{n} \rightarrow \mathbf{R}$ if and only if the differential 2-form $d q \wedge d f(q)$ is identically zero, which amounts to saying that the differential 2-form $\omega_{\mathbf{R}^{n}}=d q \wedge d p$ induces the null form on the graph $L$ of $f:$ in modern jargon [2], $L$ is a Lagrangian submanifold of the symplectic structure $\omega_{\mathbf{R}^{n}}$. The primitive $\varphi$ of $f$ is unique up to a constant, and the relation $L=d \varphi\left(\mathbf{R}^{n}\right)$ can be expressed by saying that $\varphi$ is a generating function of the Lagrangian submanifold $L$.

Let now $h: T^{*} \mathbf{R}^{n} \rightarrow T^{*} \mathbf{R}^{n}$ be a symplectic (or canonical) transformation, i.e. a diffeomorphism such that $h^{*} \omega_{\mathbf{R}^{n}}=\omega_{\mathbf{R}^{n}}$ (if $n=1$, it means that $h$ is orientation- and area-preserving). If the points of $\left(T^{*} \mathbf{R}^{n}\right)^{2}$ are denoted by $((q, p),(Q, P))$, this amounts to saying that the 2-form $d Q \wedge d P-d q \wedge d p$ induces the null 2-form on the graph $\Gamma$ of $h$. Therefore, if we introduce the isomorphism $A$ of $\left(T^{*} \mathbf{R}^{n}\right)^{2}$ onto $T^{*}\left(T^{*} \mathbf{R}^{n}\right)$ defined by $A((q, p),(Q, P)):=((Q, p),(P, q))$, the transformation $h$ is symplectic if and only if $A \Gamma$ is Lagrangian. Now, when $h$ is (for example) $C^{1}$-close enough to the identity, $A \Gamma$ is the graph of a map $F: T^{*} \mathbf{R}^{n} \rightarrow\left(T^{*} \mathbf{R}^{n}\right)^{*}$. Hence there exists a real function $\Phi(Q, p)$, unique up to a constant, such that $F=d \Phi$. In other words, $\Gamma$ is defined by the equations

$$
\left\{\begin{array}{l}
q=\partial_{p} \Phi(Q, p) \\
P=\partial_{Q} \Phi(Q, p) .
\end{array}\right.
$$

We call $\Phi$ a generating function of $h$.

However, there exist Lagrangian submanifolds of $T^{*} \mathbf{R}^{n}$ which project badly on $\mathbf{R}^{n}$ and symplectic transformations of $T^{*} \mathbf{R}^{n}$ which do not have a generating function in the above sense. Such objects appear in the study of the simplest non-linear first order partial differential equations; for example, by Corollary 3 above, the solutions of the Cauchy problem

$$
\left\{\begin{array}{l}
\partial_{t} u(t, q)+\frac{1}{2} \partial_{q} u(t, q)^{2}=0 \\
u(0, q)=u_{0}(q),
\end{array}\right.
$$

where $u_{0}$ is a real function in one variable, are obtained locally as follows: the graph of the differential of $u_{t}: q \mapsto u(t, q)$ must be $L_{t}=g_{t}\left(L_{0}\right)$, where $g_{t}(q, p)=(q+t p, p)$. Now, the reader can check that in general, all the $L_{t}$ 's are not globally graphs of functions $\left({ }^{6}\right)$.

According to the situation considered, $L_{t}$ will or will not be an acceptable solution of the problem $\left({ }^{7}\right)$. Anyway, the Lagrangian submanifolds which are not graphs have enough interest to justify an extension of the notion of a generating function $\left({ }^{8}\right)$. The same question arises for symplectic transformations far away from the identity.

$\left({ }^{6}\right)$ When $u_{0}$ varies too much, it may happen that none of them is for $t \neq 0$.

$\left({ }^{7}\right)$ It is acceptable in optics but not when the objects under study, e.g. cars, can collide.

$\left({ }^{8}\right)$ All the more since the geometric substratum of the theory of linear differential operators [29], [23] is nothing but the study of non-linear first order partial differential equations. 
2.2. Generating families. The answer to this question often is so simple that it could have been given one century sooner: just "add (finitely many) variables" and consider generating families (also called generating phase functions). Thus, as often in algebraic geometry and singularity theory, "singular" objects are viewed as sections of "regular" higher-dimensional objects. The idea first appeared at the local level (for example in Hörmander [22]), but we shall now describe a global construction.

If $\left(g_{t}\right)_{0 \leq t \leq 1}$ is a symplectic isotopy of $T^{*} \mathbf{R}^{n}$ (i.e. $(t, x) \mapsto g_{t}(x)$ is "smooth enough" and $t \mapsto g_{t}$ is a path in the group of symplectic transformations, such that $g_{0}=\mathrm{Id}$ ), it can be obtained by solving for each $x \in T^{*} \mathbf{R}^{n}$ the Cauchy problem

$$
\left\{\begin{array}{l}
\frac{d}{d t} g_{t}(x)=\dot{g}_{t}\left(g_{t}(x)\right) \\
g_{0}(x)=x
\end{array}\right.
$$

where the infinitesimal generator $\left(\dot{g}_{t}\right)_{0 \leq t \leq 1}$ of the isotopy is the time-dependent vector field defined by the first equation. When, for example, the Lipschitz constants Lip $\dot{g}_{t}$, $1 \leq t \leq 1$, are bounded from above, it is easy to check that there exists a subdivision $0=t_{0}<t_{1}<\ldots<t_{N+1}=1$ of $[0,1]$ such that, for $0 \leq j \leq N$, the symplectic transformation $h_{j}=g_{t_{j+1}} \circ g_{t_{j}}^{-1}$ is close enough to the identity to have a generating function $\Phi_{j}$. If $\Phi:\left(T^{*} \mathbf{R}^{n}\right)^{N+1} \rightarrow \mathbf{R}$ is defined by

$$
\Phi\left(\left(Q_{j}, p_{j}\right)_{0 \leq j \leq N}\right)=\sum_{j=0}^{N} \Phi_{j}\left(Q_{j}, p_{j}\right)-\sum_{j=0}^{N-1} p_{j+1} Q_{j},
$$

then, setting

we have

$$
\left\{\begin{array}{l}
q_{j}=\partial_{p} \Phi\left(Q_{j}, p_{j}\right) \\
P_{j}=\partial_{Q} \Phi\left(Q_{j}, p_{j}\right)
\end{array}\right.
$$

$$
d \Phi=P_{N} d Q_{N}+q_{0} d p_{0}+\sum_{j=0}^{N-1}\left(\left(P_{j}-p_{j+1}\right) d Q_{j}+\left(q_{j+1}-Q_{j}\right) d p_{j+1}\right) .
$$

The graph of $g_{1}=h_{N} \circ \ldots \circ h_{0}$ is in bijection with the set of those $\left(\left(q_{j}, p_{j}\right),\left(Q_{j}, P_{j}\right)\right)_{0 \leq j \leq N}$ in the product of the graphs of the $h_{j}$ 's such that $\left(q_{j+1}, p_{j+1}\right)=\left(Q_{j}, P_{j}\right)$ for $0 \leq j<j$. Hence (6), together with the fact that $\Phi_{j}$ generates $h_{j}$ for each $j$, shows that the graph of $g_{1}$ is in bijection with the set $\Sigma_{\Phi}$ of those $(Q, p ; v)=\left(Q_{N}, p_{0} ;\left(Q_{j}, p_{j+1}\right)_{0 \leq j<N}\right)$ which satisfy $\left({ }^{9}\right)$

$$
\partial_{v} \Phi(Q, p ; v)=0
$$

the bijection being

$$
\Sigma_{\Phi} \ni(Q, p ; v) \stackrel{I_{\Phi}}{\longmapsto}\left(\left(\partial_{p} \Phi(Q, p ; v), p\right),\left(Q, \partial_{Q} \Phi(Q, p ; v)\right)\right) .
$$

The function $\Phi$ is called a generating phase function of the symplectic transformation $g_{1}$; this includes the following two features:

- the map $\partial_{v} \Phi$ is transversal to 0 ( since $\left(Q_{j}, p_{j}\right)$ is a coordinate system on the graph of $h_{j}$ for each $j$ ) and therefore $\Sigma_{\Phi}$ is a $2 n$-dimensional submanifold;

- the map $I_{\Phi}$ is (for the same reason) an embedding.

$\left({ }^{9}\right)$ "Messing up" a bit the order of the variables on which $\Phi$ depends. 
The formula (6) shows that $S(Q ; v)=\Phi(Q, 0 ; v)$ is a generating phase function of the Lagrangian submanifold $L_{1}:=g_{1}(\{p=0\})$ :

- the map $\partial_{v} S$ is transversal to 0 and therefore $\Sigma_{S}:=\partial_{v} S^{-1}(0)$ is an $n$-dimensional submanifold;

- the map $\Sigma_{S} \ni(Q ; v) \stackrel{I_{S}}{\longmapsto}\left(Q, \partial_{Q} S(Q ; v)\right)$ is an embedding, whose image is $L_{1}$.

The "bad points" of $L_{1}$ can then be studied by applying singularity theory to the family $S_{Q}$ of functions of $v$ defined by $S_{Q}(v)=S(Q ; v)$ : since the family has "nothing special" in general from the viewpoint of local singularities, this is just the theory of singularities of Lagrangian and Legendrian projections, as developed by Arnold and his school [2], [4] since the mid-sixties.

2.3. Generating families which are "quadratic at infinity". With the notation of (4), the infinitesimal generator $\left(\dot{g}_{t}\right)$ of the symplectic isotopy $\left(g_{t}\right)$ satisfies $\mathcal{L}_{\dot{g}_{t}} \omega_{\mathbf{R}^{n}}=0$, i.e. $d\left(\omega_{\mathbf{R}^{n}} \dot{g}_{t}\right)=0$; hence, by Poincaré's lemma, each $\omega_{\mathbf{R}^{n}} \dot{g}_{t}$ is the differential of a real function $H_{t}$ and, if we decide for example that $H_{t}(0)=0$, then $(t, x) \mapsto H_{t}(x)$ is smooth; the family $\left(H_{t}\right)$ is called a Hamiltonian of the isotopy; the identity $\omega_{\mathbf{R}^{n}} \dot{g}_{t}=d H_{t}$ shows that $\left(H_{t}\right)$ determines $\left(\dot{g}_{t}\right)$ (which we shall denote $\dot{g}_{t}=\overrightarrow{d H_{t}}$ as in Section 1.5 above) and therefore, by (4), $\left(g_{t}\right)$. It is easy to check that the generating functions $\Phi_{j}$ in the previous paragraph are given (up to a constant) by

$$
\Phi_{j}(Q, p)=\Phi_{j}^{H}(Q, p):=p q+\int_{t_{j}}^{t_{j+1}}\left(p_{j}(t) \dot{q}_{j}(t)-H_{t}\left(q_{j}(t), p_{j}(t)\right)\right) d t
$$

where $\left(q_{j}(t), p_{j}(t)\right)=g_{t} \circ g_{t_{j}}^{-1}(q, p)$ and $Q=q_{j}\left(t_{j+1}\right)$ : as $(q, p) \mapsto(Q, p)$ is a diffeomorphism by the very definition of a generating function, this makes sense.

Consider the following three cases:

1. There exists a co-compact lattice $Z \subset T^{*} \mathbf{R}^{n}$ such that every $H_{t}$ is $Z$-periodic and therefore induces a function on the $2 n$-torus $T=T^{*} \mathbf{R}^{n} / Z$.

2. Every $H_{t}$ is $\mathbf{Z}^{n}$-periodic with respect to $q$ and all the $H_{t}$ 's, viewed as functions on $T^{*} \mathbf{T}^{n}=\mathbf{T}^{n} \times \mathbf{R}^{n *}$, vanish off the same compact subset.

3. There exists a quadratic form $K$ on $T^{*} \mathbf{R}^{n}$ such that all the $H_{t}$ 's equal $K$ off the same compact subset.

The following lemma is easy:

LEMMA 8. Let $\Phi^{H}$ denote the generating family $\Phi$ defined in the previous paragraph (with $\Phi_{j}:=\Phi_{j}^{H}$ ), and let $\Phi^{0}, \Phi^{K}$ be the generating families obtained from the same subdivision $\left(t_{j}\right)$ by replacing each $H_{t}$ by $0, K$ respectively. Then, $\Phi^{H}$ is quadratic at infinity in the following sense:

i) $\Phi^{0}, \Phi^{K}$ are quadratic forms;

ii) in cases 1 and $2, d\left(\Phi^{H}-\Phi^{0}\right):\left(T^{*} \mathbf{R}^{n}\right)^{N+1} \rightarrow\left(\left(T^{*} \mathbf{R}^{n}\right)^{N+1}\right)^{*}$ is bounded;

iii) in case $3, d\left(\Phi^{H}-\Phi^{K}\right):\left(T^{*} \mathbf{R}^{n}\right)^{N+1} \rightarrow\left(\left(T^{*} \mathbf{R}^{n}\right)^{N+1}\right)^{*}$ is bounded. 
Let us explain how this property can be used: if we make the linear change of variables $(Q, p ; v) \mapsto(Q, p ; w)$, where $w=\left(y_{j}, x_{j}\right)_{1 \leq j \leq N} \in\left(T^{*} \mathbf{R}^{n}\right)^{N}$ is defined by

$$
\left\{\begin{array}{l}
y_{j}:=Q_{j}-Q_{j-1} \quad\left(\text { recall that } Q_{N}=Q\right) \\
x_{j}:=p_{j}-p,
\end{array}\right.
$$

then $\Phi^{H}, \Phi^{0}$ and $\Phi^{K}$, viewed as functions of $(Q, p ; w)$, remain generating phase functions of $g_{1}$, Id and $\exp \overrightarrow{d K}$ respectively and we have that $\Phi^{0}(Q, p ; w)=p Q+F^{0}(w)$ with

$$
F^{0}(w)=x_{1} y_{1}+\ldots+x_{N} y_{N} .
$$

- In case $1, F^{H}(Q, p ; w):=\Phi^{H}(Q, p ; w)-p Q$ is $Z$-periodic with respect to $(Q, p)$ and therefore induces a function on $T \times\left(T^{*} \mathbf{R}^{n}\right)^{N}$ (this is the main reason for changing $v$ into $w)$; by Lemma 8 ii), the differential of $F^{H}(Q, p ; w)-F^{0}(w)$ is bounded; as $F^{0}$ is a non-degenerate quadratic form, standard arguments in differential topology show that $F^{H}$, viewed as a function on $T \times\left(T^{*} \mathbf{R}^{n}\right)^{N}$, has at least $2 n+1$ critical points, and at least $2^{2 n}$ if they are counted with their multiplicity. In other words, $g_{1}$ has at least $2 n+1$ fixed points up to translation in $Z$, and at least $2^{2 n}$ if they are counted with their multiplicity. This is the celebrated Conley-Zehnder theorem [16]: the endpoint of a Hamiltonian isotopy of a standard symplectic structure on the $2 n$-torus has at least $2 n+1$ fixed points, and at least $2^{2 n}$ if they are counted with their multiplicity.

- In case 2 , similarly, $S^{H}(Q ; w):=\Phi^{H}(Q, 0 ; w)$ is $\mathbf{Z}^{n}$-periodic with respect to $Q$ and therefore induces a function on $\mathbf{T}^{n} \times\left(T^{*} \mathbf{R}^{n}\right)^{N}$ which (as $F^{0}$ is non-degenerate) has at least $n+1$ critical points, and at least $2^{n}$ if they are counted with their multiplicity. This proves another of Arnold's conjectures [1], implying the Conley-Zehnder theorem [7]: the image of the zero section $\{p=0\}$ of $T^{*} \mathbf{T}^{n}$ by the endpoint of a Hamiltonian isotopy has at least $n+1$ intersection points with the zero section, and at least $2^{n}$ if the intersection is transversal.

- In case 3, assume that the quadratic form $F^{K}(Q, p ; w)=\Phi^{K}(Q, p ; w)-p Q$ is nondegenerate (which means that the linear Hamiltonian vector field $\overrightarrow{d K}$ has no non-trivial periodic orbit of period 1). Then, the same arguments show that $F^{H}$ has at least one critical point, i.e. that $g_{1}$ has at least one fixed point. For suitable $H$, one can use this approach to give a very simple proof [10], [27] of the Weinstein conjecture in $\mathbf{R}^{2 n}[33]$ and construct the Hofer-Zehnder capacity [21].

2.4. Quasi-functions and their generating families. In case 2 above, a symplectic version [7] of Thom's isotopy extension lemma [32] implies the following: a smooth deformation $\left(L_{t}\right)_{0 \leq t \leq 1}$ of the zero section $L_{0}=\{p=0\}$ of $T^{*} \mathbf{T}^{n}$ is of the form $L_{t}=h_{t}\left(L_{0}\right)$ for a Hamiltonian isotopy $\left(h_{t}\right)$ of $T^{*} \mathbf{T}^{n}$ (meaning that $\omega_{\mathbf{T}^{n}} \dot{h}_{t}$ is exact for every $t$ ) if and only if the Liouville form $p d q$ of $T^{*} \mathbf{T}^{n}$ induces an exact 1-form on each $L_{t}$, and the same is true when $\mathbf{T}^{n}$ is replaced by an arbitrary compact manifold $M$.

Now, this condition means that each $L_{t}$ is the image of a Legendrian submanifold $\tilde{L}_{t}$ of $J^{1} M$ (unique up to translation in the $u$ direction) by the canonical projection $\pi:(q, u, p) \mapsto(q, p)$. In fact, if $\left(H_{t}\right)$ is a Hamiltonian of $\left(h_{t}\right)$ we can lift $\left(h_{t}\right)$ to the contact isotopy $\left(\tilde{h}_{t}\right)$ with (contact) Hamiltonian $\left(H_{t} \circ \pi\right)$, choose for $\tilde{L}_{0}$ the zero section (image $\{u=0, p=0\}$ of $\left.j^{1} 0\right)$ and let $\tilde{L}_{t}=\tilde{h}_{t}\left(\tilde{L}_{0}\right)$. 
Quasi-functions and their critical points. Following Arnold [3], this is expressed by calling each $\tilde{L}_{t}$ a quasi-function; since the isotopy extension lemma is true in contact geometry, a quasi-function on $M$ can also be defined as a Legendrian submanifold of $J^{1} M$ which can be obtained from the zero section by a smooth deformation through Legendrian submanifolds of $J^{1} M$.

The 1-jet of a true function $\varphi$ is a quasi-function, for it can be joined to the zero section by the smooth family $j^{1}(t \varphi)(M)$; hence it is natural to define the critical points of a quasi-function to be its intersections with $\{p=0\}$, and to call them non-degenerate when the intersection is transversal.

As the restriction of the projection $\pi: J^{1} M \rightarrow T^{*} M$ to a quasi-function is a Lagrangian immersion but not necessarily an embedding, the following result is more general than its symplectic version, due to Sikorav [30] (and established above if $M=\mathbf{T}^{n}$ ):

THEOREM 9 ([15]). For each quasi-function $L$ on a closed manifold $M$, there exists a finite-dimensional vector space $E$ and a real function $S$ on $M \times E$ with the following properties:

1. $S$ is a generating family for $L$ :

- the "vertical derivative" $M \times E \ni(Q ; v) \mapsto \partial_{v} S(Q ; v) \in E^{*}$ is transversal to 0 , hence $\Sigma_{S}:=\partial_{v} S^{-1}(0)$ is a submanifold of the same dimension as $M$.

- $\Sigma_{S} \ni(Q ; v) \stackrel{J_{S}}{\longmapsto}\left(Q, S(Q ; v), \partial_{Q} S(Q ; v)\right)$ is an embedding and $J_{S}\left(\Sigma_{S}\right)=L$.

2. $S$ is "non-degenerate quadratic at infinity" in the following sense: there exists a non-degenerate quadratic form $K$ on $E$ such that $M \times E \ni(Q ; v) \mapsto S(Q ; v)-K(v)$ has compact support.

More precisely, if $\left(L_{t}\right)_{0 \leq t \leq 1}$ is a smooth family of Legendrian submanifolds joining the zero section $L_{0}$ to $L_{1}=L$, there exists a smooth real function $(t, Q ; v) \mapsto S_{t}(Q ; v)$ on $[0,1] \times M \times E$ such that $S_{t}$ is a generating family of $L_{t}$ for every $t$, quadratic at infinity in the sense of Property 2 above (with the same $K$ for every $t$ ).

By Property 1 , the critical points of $L$ are the images by $J_{S}$ of the critical points of $S$; hence Property 2 implies at once the following result, more general than the symplectic version due to Hofer [20]:

COROLlary 10. The number of critical points of a quasi-function on a closed manifold $M$ is bounded below by the cuplength $c(M)$ of $M$ plus 1 , and by the sum $\mathrm{b}_{*}(M)$ of the Betti numbers of $M$ when none of those critical points is degenerate.

In other words, the lower bounds for the number of critical points of a "generalised function" (quasi-function) on $M$ are essentially the same as for real functions on $M$.

About the proof of Theorem 9. Far from Sikorav's "intrinsic" proof in [30], Chekanov had the idea of embedding $M$ as a submanifold of $\mathbf{R}^{n}$. Theorem 9 follows by extending $\left(L_{t}\right)$ to a "suitable" deformation $\left(\tilde{L}_{t}\right)$ of the zero section in $J^{1} \mathbf{R}^{n}$ : by the composition formula (5) [15], [19] (or its generalisation to contact transformations [11]), $\tilde{L}_{t}$ has a generating family $\tilde{S}_{t}$, defined on $\mathbf{R}^{n} \times E$, and $S_{t}$ is just its restriction to $M \times E$ (such is the meaning of "suitable"), modulo some "cut-off" using bump functions, necessary because 
of our new and rather demanding notion of "quadratic non-degenerate at infinity", which is not essential.

2.5. Application to classical Cauchy problems. Given a manifold $M$ (which we assume closed for simplicity) and a smooth family $\left(H_{t}\right)_{0 \leq t \leq 1}$ of real functions on $J^{1} M$, we are looking for a solution of the Hamilton-Jacobi equation

$$
\partial_{t} u+H_{t}\left(q, u, \partial_{q} u\right)=0
$$

which equals a given (smooth) $\varphi_{0}: M \rightarrow \mathbf{R}$ for $t=0$. Denoting by $\left(g_{t}\right)$ the (local) contact isotopy generated by the Lie field with Hamiltonian $H_{t}$, we assume that the quasi-function $L_{t}:=g_{t}\left(j^{1} \varphi_{0}(M)\right)$ is well-defined for every $t$ (which is the case when $\left(H_{t}\right)$ has compact support, for example). By Corollary 3 above, if our problem admits a smooth solution $\varphi:(t, x) \mapsto \varphi_{t}(x)$, then it is given by the formula $j^{1} \varphi_{t}(M)=L_{t}$.

Since $M$ is closed, this formula does define a genuine solution of our Cauchy problem for small enough $t$. When $t$ increases, we have seen that, if $\pi_{M}$ denotes the natural projection of $J^{1} M$ onto $J^{0} M=M \times \mathbf{R}$, the Cerf diagram (wavefront, according to Arnold)

$$
W:=\bigcup_{0 \leq t \leq 1}\left(\{t\} \times \pi_{M}\left(L_{t}\right)\right)
$$

may not be the graph of a function $(t, q) \mapsto \varphi_{t}(q)$, but a singular hypersurface, which appears globally as a discriminant because of the following consequence of Theorem 9:

LEMMA 11. Under the above hypotheses, there exist a finite-dimensional vector space $E$, a smooth real function $S:(t, Q ; v) \mapsto S_{t}(Q ; v)$ on $[0,1] \times M \times E$ and a nondegenerate quadratic form $K$ on $E$ with the following properties:

1. For $0 \leq t \leq 1, S_{t}$ is a generating family for $L_{t}$.

2. The function $[0,1] \times M \times E \ni(Q ; v) \mapsto S_{t}(Q ; v)-K(v)$ has compact support.

Proof. Apply Theorem 9 to the deformation $\left(h\left(L_{t}\right)\right)$ of the zero section, where $h$ is the contact transformation of $J^{1} M$ given by $h(q, u, p)=\left(q, u-\varphi_{0}(q), p-d \varphi_{0}(q)\right)$ and denote by $S^{\prime}$ the generating family thus obtained. Then the function $(Q ; v) \mapsto \varphi_{0}(Q)+S_{t}^{\prime}(Q ; v)$ is a generating family of $L_{t}$ for each $t$, which almost satisfies our requirements: to get Property 2 stricto sensu, just use bump functions.

It does follows that the wavefront $W$ is the discriminant of $S$, viewed as a family of functions of $v \in E$ depending on the parameter $(t, Q) \in[0,1] \times M$. In some cases (caustics), this wavefront is the "physical" solution of the problem.

When, on the contrary, the only acceptable solutions are functions or distributions on $M$, Lemma 11 enables us to construct such a solution as follows: let $C>0$ be large, so that every $S_{t, Q}: v \mapsto S_{t}(Q ; v)$ coincides with $K$ on $E_{C}:=S_{t, Q}^{-1}(-\infty,-C]=$ $K^{-1}(-\infty,-C]$. For each $(t, Q)$, consider the "minimax" critical value of $S_{t, Q}$ defined by

$$
\varphi(t, Q)=\varphi_{t}(Q):=\inf _{\sigma} \max S_{t, Q} \circ \sigma,
$$

where $\sigma$ varies among all relative singular homology cycles whose class is a fixed generator of $H_{j}\left(E, E_{C}\right) \cong \mathbf{Z}$ and $j$ is the index of $K$. It is quite easy to check that $\varphi$ is a Lipschitzian solution of our problem (this was suggested to me [9], [11] by Sikorav); 
indeed, its definition implies that it is a Lipschitzian section of the wavefront-hence $\varphi_{t}$ is smooth and satisfies $j^{1} \varphi_{t}(M)=L_{t}$ whenever the latter is well-projected, for example when $t=0$; moreover, the 1 -jet of $\varphi$ in the sense of distributions is its almost everywhere defined 1 -jet in the usual sense, which is readily seen to satisfy (7).

This generalised solution in the sense of analysis, obtained as a section of a generalised solution in the sense of geometry, does not depend on the choice of $S$ because of the following result, first stated ([34], Proposition 1.5) in the symplectic case:

Theorem 12 ([31]). If $S_{1}: M \times E_{1} \rightarrow \mathbf{R}$ and $S_{2}: M \times E_{2} \rightarrow \mathbf{R}$ are two generating families of the same quasi-function on $M$, quadratic non-degenerate at infinity, they are stably (right-)equivalent: there exist non-degenerate quadratic forms $K_{j}$ on finitedimensional real vector spaces $F_{j}(j=1,2)$, such that $S_{1}\left(Q ; v_{1}\right)+K_{1}\left(w_{1}\right)=S_{2}\left(Q ; v_{2}\right)+$ $K_{2}\left(w_{2}\right)$ for some diffeomorphism $\left(Q ; v_{1}, w_{1}\right) \mapsto\left(Q ; v_{2}\left(Q ; v_{1}, w_{1}\right), w_{2}\left(Q ; v_{1}, w_{1}\right)\right)$.

Until recently, we did not know how to characterise the minimax solution, except in one case [24]: when every $H_{t}$ is strictly convex with respect to $p, \varphi_{t}(Q)$ is just the $u$-coordinate of the lowest point of the wavefront above $(t, Q)$; indeed, $L_{t}$ admits a generating family which is positive definite at infinity, hence the minimax is a minimum. This implies at once that $\varphi$ satisfies the "entropy condition" [25], [18] or, in more recent language, is a "viscosity solution".

In the general case, Gianmarco Capitanio has just obtained [5] the direct geometric characterisation of the minimax solution we were looking for.

The advantage of this solution is that its generic singularities can be studied using singularity theory, yielding for example the following result:

TheOREM 13 ([24]). A generic minimax function $\varphi$ on a manifold $B$ is smooth except possibly along a closed stratified hypersurface; viewed in appropriate smooth local charts of $B$, this hypersurface and the graph of $\varphi$ are semi-algebraic.

It would certainly be good to have an analogue of this theorem for viscosity solutions, which most applied mathematicians seem to find interesting even though they are not as appealing aesthetically as minimax solutions.

Final Note. René Thom died while I was writing this paper, which is a pale reflection of his enormous influence on science - and, at a more anecdotal level, on my destiny. The construction of weak solutions of Hamilton-Jacobi equations below can be viewed as an answer to a question he repeatedly asked his seminar in the mid-seventies.

\section{References}

[1] V. I. Arnol'd, Sur une propriété topologique des applications globalement canoniques de la mécanique classique, C. R. Acad. Sci. Paris 261 (1965), 3719-3722.

[2] V. I. Arnol'd, On a characteristic class entering into conditions of quantization, Funktsional. Anal. i Prilozhen. 1 (1967), 1-14 (Russian); English transl.: Funct. Anal. Appl. 1 (1967), $1-13$.

[3] V. I. Arnol'd, First steps of symplectic topology, Uspekhi Mat. Nauk 41:6 (1986), 3-18 (Russian); English transl.: Russian Math. Surveys 41:6 (1986), 1-21. 
[4] V. I. Arnol'd, S. M. Guseŭn-Zade, A. N. Varchenko, Singularities of Differentiable Maps I, Monogr. Math. 82, Birkhäuser, Boston, 1985.

[5] G. Capitanio, Caractérisation géométrique des solutions de minimax pour l'équation de Hamilton-Jacobi, Enseign. Math. (2) 49 (2003), 3-24.

[6] M. Chaperon, Quelques outils de la théorie des actions différentiables, Astérisque 107-108 (1983), 259-275.

[7] M. Chaperon, Quelques questions de géométrie symplectique (d'après, entre autres, Poincaré, Arnold, Conley et Zehnder), Séminaire Bourbaki, Astérisque 105-106 (1983), 231-249.

[8] M. Chaperon, Géométrie différentielle et singularités de systèmes dynamiques, Astérisque 138-139 (1986).

[9] M. Chaperon, Lois de conservation et géométrie symplectique, C. R. Acad. Sci. Paris Sér. I Math. 312 (1991), 345-348.

[10] M. Chaperon, Familles génératrices, Lectures given in 1990 at a summer school in Samos, Erasmus Publication, University of Thessaloniki (1993). Can be found on the author's web site http://www.math.jussieu.fr/ chaperon/

[11] M. Chaperon, On generating families, in: The Floer Memorial Volume, H. Hofer et al. (eds.), Progr. Math. 133, Birkhäuser, Basel, 1995, 283-296.

[12] M. Chaperon, A remark on Liouville vector fields and a theorem of Manouchehri, Ergodic Theory Dynam. Systems 19 (1999), 895-899.

[13] M. Chaperon, Invariant manifolds revisited, Tr. Mat. Inst. Steklova 236 (2002), Differ. Uravn. i Din. Sist., 428-446 (Russian); English transl.: Proc. Steklov Inst. Mat. 236 (2002), 415-433.

[14] M. Chaperon, Stable manifolds and the Perron-Irwin method, Ergodic Theory Dynam. Systems, to appear in the volume dedicated to the memory of Michael R. Herman.

[15] Yu. V. Chekanov, Critical points of quasi-functions and generating families of legendrian manifolds, Funktsional. Anal. i Prilozhen. 30:2 (1996), 56-69 (Russian); Funct. Anal. Appl. 30 (1996), 118-128.

[16] C. C. Conley, E. Zehnder, The Birkhoff-Lewis fixed point theorem and a conjecture of V. I. Arnol'd, Invent. Math. 73 (1983), 33-49.

[17] A. Davydov, E. Rosales-González, Smooth normal forms of folded resonance saddles and nodes and complete classification of generic linear second order PDE's on the plane, in: International Conference on Differential Equations (Lisboa, 1995), World Sci. Publishing, River Edge, 1998, 59-68.

[18] T. Debeneix, Condition d'entropie pour certains systèmes hyperboliques quasilinéaires de $n$ équations à $n$ variables d'espace, C. R. Acad. Sci. Paris 287 (1978), Sér. A-B, A763-A766.

[19] Y. Eliashberg, M. Gromov, Lagrangian intersection theory: finite-dimensional approach, in: Geometry of Differential Equations, Amer. Math. Soc. Transl. Ser. 2, 186, Amer. Math. Soc., Providence, 1998, 27-118.

[20] H. Hofer, Lagrangian embeddings and critical point theory, Ann. Inst. H. Poincaré Anal. Non Linéaire 2 (1985), 407-462.

[21] H. Hofer, E. Zehnder, Symplectic Invariants and Hamiltonian Dynamics, Birkhäuser, Basel, 1994.

[22] L. Hörmander, Fourier Integral Operators I, Acta Math. 127 (1971), 79-183.

[23] L. Hörmander, The Analysis of Linear Partial Differential Operators, Springer, Berlin, 1983-85. 
[24] T. Joukovskaïa, Singularités de minimax et solutions faibles d'équations aux dérivées partielles, Thèse, Université Paris 7, January 1994.

[25] P. D. Lax, Hyperbolic systems of conservation laws II, Comm. Pure Appl. Math. 10 (1957), $537-566$.

[26] M. Manouchehri, Formes normales d'équations différentielles implicites et de champs de Liouville, Ergodic Theory Dynam. Systems 16 (1996), 779-789.

[27] D. McDuff, D. Salamon, Introduction to Symplectic Topology, Oxford Math. Monogr., The Clarendon Press, Oxford Univ. Press, New York, 1995. Second edition, 1998.

[28] J. Moser, On the volume elements on a manifold, Trans. Amer. Math. Soc. 120 (1965), 286-294.

[29] M. Sato, T. Kawai, M. Kashiwara, Microfunctions and pseudo-differential equations, in: Hyperfunctions and Pseudo-Differential Equations, Lecture Notes in Math. 287, Springer, Berlin, 1973, 265-529.

[30] J.-C. Sikorav, Problèmes d'intersections et de points fixes en géométrie hamiltonienne, Comment. Math. Helv. 62 (1987), 62-73.

[31] D. Théret, A complete proof of Viterbo's uniqueness theorem on generating functions, Topology Appl. 96 (1999), 249-266.

[32] R. Thom, La classification des immersions, Séminaire Bourbaki 1957-58, exposé 157.

[33] C. Viterbo, A proof of Weinstein's conjecture in $\mathbf{R}^{2 n}$, Ann. Inst. H. Poincaré Anal. Non Linéaire 4 (1987), 337-356.

[34] C. Viterbo, Symplectic topology as the geometry of generating functions, Math. Ann. 292 (1992), 685-710. 